\title{
A NEW APPROACH TO TERMINAL SLIDING MODE CONTROL DESIGN
}

\author{
Yiguang Hong, Guowu Yang, Daizhan Cheng, and Sarah Spurgeon
}

\begin{abstract}
In this paper, terminal sliding mode control design is considered. A control method, different from many existing terminal sliding model control design methods, is proposed based on a new switching law and continuous finite-time control ideas. Then terminal sliding mode control laws are constructed for some classes of nonlinear systems.
\end{abstract}

KeyWords: Terminal sliding mode control, finite-time convergence, nonsmooth feedback.

\section{INTRODUCTION}

Sliding mode control has been studied for many decades and is now one of the most active areas of research on nonlinear control theory. Many reports on sliding mode control and its extensions can be found in the literature, including [1-4]. In recent years, an increasing interest has focused on terminal sliding mode control, with the goal of rendering finite-time convergence to equilibrium of the closed-loop system in question [5-7]. However, these control methods may cause singularity problems and influence the terminal sliding surfaces. In fact, [8] proposed a twophase control scheme to avoid the singularity in their original control law.

The main contribution of this paper is as follows. Different from many existing recursive design procedures for terminal sliding modes, we propose a design method that combines a new switching scheme with some ideas related to continuous finite-time control given in $[9,10]$. The constructed terminal sliding mode controller is not in a recur-

Manuscript received November 13, 2002; revised April 12, 2004; accepted May 13, 2004.

Yiguang Hong and Daizhan Cheng are with Institute of Systems Science, Chinese Academy of Sciences, Beijing 100080, China (e-mail: yghong@iss03.iss.ac.cn).

Guowu Yang is with Department of Electrical and Computer Engineering, Portland State University, Portland, OR 97207-0751, U.S.A.

Sarah Spurgeon is with Department of Engineering, University of Leicester, University Road, Leicester LE1 7RH, U.K. sive form and removes singularities outside of sliding surfaces.

The rest of the paper is organized as follows. In section 2, the problem formulation is presented, while some theoretic analysis is given in section 3 . Then, in section 4 , terminal sliding mode control laws are built for two classes of systems. Finally, concluding remarks are given in section 5.

\section{PROBLEM FORMULATION}

In this paper, we focus on a single-input control system of the form

$$
\left\{\begin{array}{l}
\dot{x}_{1}=f_{1}(x) \\
\cdots \\
\dot{x}_{n-1}=f_{n-1}(x) \\
\dot{x}_{n}=f_{n}(x)+u
\end{array}\right.
$$

with $x=\left(x_{1}, \ldots, x_{n}\right)^{T} \in \mathcal{R}^{n}, f_{i}(x)$ smooth, and $f_{i}(0)=0$ for $i=$ $1, \ldots, n$.

Let $s: \mathcal{R}^{n} \rightarrow \mathcal{R}$ be a continuous function with $s(0)=0$. The sliding surface is defined as $S=\left\{x \in \mathcal{R}^{n}: s(x)=0\right\}$. The sliding mode design procedure can be described roughly as consisting of two main steps. First, a sliding surface satisfying $\frac{\partial s}{\partial x_{n}} \neq 0$ is found such that $s(x(t)) \equiv 0$ implies $x(t) \rightarrow$ 
0 as $t \rightarrow \infty$; then, a stabilizing feedback law is constructed in the form

$$
u(x)=\left\{\begin{array}{llc}
u^{+}(x), & \text { if } & s>0 \\
u^{-}(x), & \text { if } & s<0
\end{array} .\right.
$$

Most sliding mode control laws make the controlled system (1) convergent to the sliding surfaces in finite time; then, along the sliding surfaces, the systems converge to the equilibrium $x=0$ of system (1) as time goes to infinity. However, terminal sliding mode control laws can achieve more by steering the states to equilibrium in finite time.

Definition 1. For system (1), $u=u(x)$ is called a finite-time convergent controller if the equilibrium $x=0$ of the closedloop system (1) under this feedback law is finite-time convergent; that is, for any initial condition $x(0)=x^{0} \in \mathcal{R}^{n}$, there is a settling time $T \geq 0$, which satisfies

$$
\lim _{t \rightarrow T} x\left(t ; 0, x^{0}\right)=0,
$$

and

$$
x\left(t ; 0, x^{0}\right)=0, \quad \text { if } \quad t>T
$$

for every solution $x\left(t ; 0, x^{0}\right)$ to the closed-loop system (1). Moreover, if the controller is also a sliding mode controller, then it is called a terminal sliding mode controller. form:

In this paper, we assume that $s(x)$ takes the following

$$
s(x)=x_{n}-h\left(x_{1}, \ldots, x_{n-1}\right), \quad h(0, \ldots, 0)=0,
$$

where $h\left(x_{1}, \ldots, x_{n-1}\right)$ is a continuous function satisfying

$$
\left|h\left(x_{1}, \ldots, x_{n-1}\right)\right|<K_{0}+L_{0} \sum_{i=1}^{n-1}\left|x_{i}\right|
$$

for some positive numbers $K_{0}$ and $L_{0}$.

Many choices of sliding modes satisfy condition (3). For example, $s(x)=\sum_{i=1}^{n-1} a_{i} x_{i}$ is a widely used form, and taking $K_{0}=1$ and $L_{0}=\max \left\{a_{i}, i=1, \ldots, n-1\right\}$ will make (3) hold.

In the following, two lemmas will be introduced for the following analysis. Their proofs are quite obvious and, therefore, omitted here.

Lemma 1. For any $0<\alpha<1$ and $M_{0}>0, M_{1}>0$ exists such that $|z|^{\alpha} \leq M_{0}+M_{1}|z|$ holds for all $z \in \mathcal{R}$.

Lemma 2. Suppose that $a, b$, and $m>1$ are all positive numbers. Then, $\left(a^{m}+b^{m}\right)^{1 / m} \leq a+b$.

\section{TERMINAL SLIDING MODE}

First, we will present results for finite-time convergence to given sliding surfaces.
Theorem 1. If the sliding surface $S$ is taken with $h(x)$ satisfying (3), then the system (1) reaches $S$ in finite time by the control law

$$
u=-f_{n}(x)+v,
$$

where

$$
v=-K\left(1+\sum_{i=1}^{n-1}\left|f_{i}(x)\right|\right) \operatorname{sgn}(s), \quad K>\max \left\{L_{0}, 1\right\}, \quad s \neq 0,
$$

and $\operatorname{sgn}(\cdot)$ is denoted as the sign function.

Proof. The task, in fact, is to prove that, for any initial condition $x(0) \neq 0$ with $s(x(0)) \neq 0$, the feedback (4) will lead to $s(x(t))=0$ in finite time.

Let $x(0)=x^{0}=\left(x_{1}^{0}, \ldots, x_{n-1}^{0}, x_{n}^{0}\right)$ be the initial condition. The trajectory with the initial condition is denoted by $x(t ; 0, x(0))$, or $x(t)$ for simplicity.

We will first study the case where $s(x(0))>0$; that is, $x_{n}^{0}>h\left(x_{1}^{0}, \ldots, x_{n-1}^{0}\right)$. We will prove that $T>0$ exists such that $s(x(T))=0$ by contradiction.

Suppose that $T>0$ does not exist such that $s(x(T))=0$. Because $s(x(0))>0$ and $s(x(t))$ is continuous, $s(x(t))>0$ for any $t>0$; that is, $x_{n}(t)>h\left(x_{1}(t), \ldots, x_{n-1}(t)\right)$ for any $t>0$. Therefore,

$\dot{x}_{n}=-K\left(1+\sum_{i=1}^{n-1}\left|f_{i}(x)\right|\right) \operatorname{sgn}(s)=-K\left(1+\sum_{i=1}^{n-1}\left|f_{i}(x)\right|\right)$. (6)

Integrating both sides of (6) gives

$$
\begin{aligned}
& x_{n}(t)-x_{n}^{0}=-K t-K \sum_{i=1}^{n-1} \int_{0}^{t}\left|f_{i}(x)\right| d t \\
& \leq-K t-K \sum_{i=1}^{n-1}\left|\int_{0}^{t} \dot{x}_{i} d t\right| \leq-K t-K \sum_{i=1}^{n-1}\left|x_{i}(t)\right|+K \sum_{i=1}^{n-1}\left|x_{i}^{0}\right|,
\end{aligned}
$$

which implies

$$
x_{n}(t) \leq-\left(K t-x_{n}^{0}-K \sum_{i=1}^{n-1}\left|x_{i}^{0}\right|\right)-K \sum_{i=1}^{n-1}\left|x_{i}(t)\right| .
$$

Recall (3), i.e., $\left|h\left(x_{1}, \ldots, x_{n-1}\right)\right|<K_{0}+L_{0} \sum_{i=1}^{n-1}\left|x_{i}\right|$. If we make $t_{1}$ large enough, say

$$
t_{1}>\frac{K_{0}+\left|x_{n}^{0}\right|}{K}+\sum_{i=1}^{n-1}\left|x_{i}^{0}\right|
$$

then we have

$$
x_{n}\left(t_{1}\right)<-K_{0}-L_{0} \sum_{i=1}^{n-1}\left|x_{i}\left(t_{1}\right)\right| \leq h\left(x_{1}\left(t_{1}\right), \ldots, x_{n-1}\left(t_{1}\right)\right) ;
$$

that is, $s\left(x\left(t_{1}\right)\right)<0$. This contradiction shows that there exists a reaching time $T_{1}<t_{1}$ such that $s\left(x\left(T_{1}\right)\right)=0$.

Similarly, if $s(x(0))<0$, that is, $x_{n}^{0}<h\left(x_{1}^{0}, \ldots, x_{n-1}^{0}\right)$, 
then there is another reaching time $T_{2}>0$ such that $s\left(x\left(T_{2}\right)\right)$ $=0$.

Thus, the assertion follows.

An important merit of sliding mode control is that it provides robustness to uncertainties. Consider system (1) with $f_{i}, i=1, \ldots, n$ uncertain. Suppose

$$
\left|f_{i}(x)\right| \leq \phi_{i}(x), \quad i=1, . ., n,
$$

where the functions $\phi_{i}(x), i=1, \ldots, n$, are known and $\phi_{i}(0)$ $=0$. Then, using an idea that is similar to the one employed in the proof of Theorem 1, we have the following theorem.

Theorem 2. If $h\left(x_{1}, \ldots, x_{n-1}\right)$ satisfies (3), then we can put the control law in the form

$$
u(x)=-K\left(1+\sum_{i=1}^{n} \phi_{i}(x)\right) \operatorname{sgn}(s), \quad s \neq 0
$$

with $K>\max \left\{K_{0}, L_{0}, 1\right\}$, such that the uncertain system (1) reaches $S$ in finite time.

In fact, the goal of terminal sliding mode control is to find a suitable sliding surface such that the state outside of the selected sliding surface is finite-time convergent to it while the system state in the sliding surface tends to equilibrium in finite time. We will sketch our design idea in the following. We first select $s(x)=x_{n}-h\left(x_{1}, \ldots, x_{n-1}\right)$ such that condition (3) holds and

$$
\left\{\begin{array}{l}
\dot{x}_{1}=f_{1}\left(x_{1}, \ldots, x_{n-1}, h\right) \\
\cdots \\
\dot{x}_{n-1}=f_{n-1}\left(x_{1}, \ldots, x_{n-1}, h\right)
\end{array}\right.
$$

is finite time convergent to the origin on $S=\left\{x \in \mathcal{R}^{n}: s(x)\right.$ $=0\}$. Then, we adopt the control laws given in the form of (4) or (8) to guarantee finite-time convergence to $S$ and force the state to move along $S$, reaching the equilibrium in finite time.

Remark 1. In many conventional cases, we can construct $u$ $=u_{e q}$ on $S=\left\{x \in \mathcal{R}^{n}: s(x) \equiv 0\right\}$ based on knowledge of $f_{i}, i=$ $1, \ldots, n$ in order to make $S$ an invariant set or even manifold; that is, $s(x(t)) \equiv 0$ for any $t \geq 0$ with the initial condition $s(x(0))=0$. However, terminal sliding mode control may result in the singularity problem for $u_{e q}$ and prevent the existence of the corresponding sliding surface $S$ that is kept invariant by $u_{e q}$ in some sense. This has been widely noted $[5,6,8]$.

\section{TWO CLASSES OF SYSTEMS}

In this section, based on the discussion in section 3, we will consider how to construct terminal sliding mode controllers for two classes of systems in form of (1).

\subsection{Class 1}

Consider the system

$$
\left\{\begin{array}{l}
\dot{x}_{1}=x_{2} \\
\cdots \\
\dot{x}_{n-1}=x_{n} \\
\dot{x}_{n}=f_{n}(x)+u
\end{array} .\right.
$$

As shown in [5,8], existing terminal sliding modes for system (10) have usually been constructed in a recursive structure as follows:

$$
s_{0}=x_{1}, \quad \ldots, \quad s_{n-1}=\dot{s}_{n-2}+b_{n-2} s_{n-2}^{q_{n-1} / p_{n-1}},
$$

where $(n-i-1) /(n-i)<q_{i+1} / p_{i+1}<1$ with $p_{i+1}, q_{i+1}, i=0, \ldots, n$ -2 as positive odd integers and $b_{i}>0, i=0, \ldots, n-2$. Then, the control law is taken as

$$
u=u_{e q}-K \operatorname{sgn}\left(s_{n-1}\right) .
$$

$u_{e q}(x)$ is given to keep $s(x(t))=0$ if $s(x(0))=0$, but this may not be satisfactory because a singularity or uncertainty may occur in the feedback law (11). In [6], a terminal sliding mode controller was given as

$$
u=-f_{n}(x)-\beta_{0} \operatorname{sgn}\left(\phi_{n-1, n}\right), \quad \beta_{0}>0
$$

with properly chosen positive parameters $\beta_{i}$, positive definite functions $N_{i, n}\left(x_{1}, \ldots, x_{i}\right), i=1, \ldots, n-1$, and a (recursive) sliding mode:

$$
\phi_{0, n}=x_{1}, \quad \ldots, \quad \phi_{i, n}=x_{i+1}+\beta_{i} N_{i, n}\left(x_{1}, \ldots, x_{i}\right) \operatorname{sgn}\left(\phi_{i-1, n}\right)
$$

for $i=1, \ldots, n-1$.

Our approach is as follows. We take $s=x_{n}-h\left(x_{1}, \ldots\right.$, $\left.x_{n-1}\right)$ such that

$$
\left\{\begin{array}{l}
\dot{x}_{1}=x_{2} \\
\ldots \\
\dot{x}_{n-2}=x_{n-1} \\
\dot{x}_{n-1}=h\left(x_{1}, \ldots, x_{n-1}\right)
\end{array}\right.
$$

is finite-time convergent to the origin. In fact, system (13) admits a continuous finite-time convergent feedback law $h=$ $v_{n-1}\left(x_{1}, \ldots, x_{n-1}\right)$ (e.g., $\left.[9,10]\right)$. Thus, in our design, we select $h=v_{n-1}$ in one of the forms provided in $[9,10]$. In this way, we make system (13) finite-time convergent to its origin on sliding surface $S$. Moreover, the condition (3) for this $h$ can also be verified using Lemmas 1 and 2 (see the following examples for some details). Then, with the control law in the form of (4) or (8), the state outside of the sliding surface is finite-time convergent to the surface $S$. Hence, system (10) with $s(x)=x_{n}-v_{n-1}$ and its corresponding control law are finite-time convergent to the origin. 
For the purpose of illustration, we will give two examples.

Example 1. Consider

$$
\left\{\begin{array}{l}
\dot{x}_{1}=x_{2} \\
\dot{x}_{2}=f_{2}(x)+u
\end{array} .\right.
$$

Take a continuous function $h\left(x_{1}\right)=-x_{1}^{3 / 5}$ and then $s=x_{2}$ $+x_{1}^{3 / 5}$. Thus, for the given $h$, (3) can be satisfied with $K_{0}=$ 3 and $L_{0}=2$, due to Lemma 1 . Then, we can take

$$
u(x)=\left\{\begin{array}{ll}
-f_{2}(x)-3\left(1+\left|x_{2}\right|\right) \operatorname{sgn}(s), & \text { if } s \neq 0 \\
u_{e q}(x)=-f_{2}(x)+\frac{3}{5} x_{1}^{1 / 5}, & \text { if } s=0
\end{array} .\right.
$$

As a result, the system converges to the sliding surface in finite time. Once it converges to the sliding surface $(s=0)$, we have $\dot{x}_{1}=-x_{1}^{3 / 5}$, which is finite-time convergent to $x_{1}$ $=0$, that is, the origin $x=0$, because of $s=0$. This directly leads to finite-time convergence of the closed-loop system.

Example 2. Consider

$$
\left\{\begin{array}{l}
\dot{x}_{1}=x_{2}, \\
\dot{x}_{2}=x_{3}, \\
\dot{x}_{3}=f_{3}(x)+u
\end{array} .\right.
$$

First, we employ the method in [5], and we have

$$
s_{0}=x_{1}, \quad s_{1}=\dot{s}_{0}+s_{0}^{7 / 9}, \quad s_{2}=\dot{s}_{1}+s_{1}^{5 / 7} .
$$

Then the control law is given as

$$
u(x)=u_{e q}(x)-K \operatorname{sgn}\left(s_{2}\right), \quad K>0
$$

with

$$
\begin{aligned}
u_{e q}(x) & =-f_{3}(x)-\left(\ddot{s}_{0}^{7 / 9}\right)-\left(\dot{s}_{1}^{5 / 7}\right) \\
& =-f_{3}(x)-\frac{7 x_{3}}{9 x_{1}^{2 / 9}}-\frac{5\left(x_{3}+\frac{7 x_{2}}{9 x_{1}^{2 / 9}}\right)}{7\left(x_{2}+x_{1}^{7 / 9}\right)},
\end{aligned}
$$

which may contain singularities.

Next, consider our proposed method for terminal sliding mode control. Take a continuous function

$$
s(x)=x_{3}+x_{1}^{\alpha_{1}}+x_{2}^{\alpha_{2}}, \quad 0<\alpha_{1}<1, \quad \alpha_{2}=\frac{2 \alpha_{1}}{1+\alpha_{1}} .
$$

with $\alpha_{i}=q_{i} / p_{i}$ and $p_{i}>0, q_{i}>0$ as suitable odd integers for $i=1,2$. For example, we take $\alpha_{1}=1 / 5$ or $\alpha_{1}=3 / 7$, and then $\alpha_{2}=1 / 3$ or $\alpha_{2}=3 / 5$. When $x \in S$ (i.e., $s(x)=0$ ), we have

$$
\left\{\begin{array}{l}
\dot{x}_{1}=x_{2} \\
\dot{x}_{2}=-x_{1}^{\alpha_{1}}-x_{2}^{\alpha_{2}}
\end{array}\right.
$$

which is finite-time convergent [1]. Moreover, for $s(x)$ given in (18), (3) can be satisfied with $K_{0}=3$ and $L_{0}=2$, due to Lemma 1 . Then, by Theorem 1 , we can construct a feedback law which is of the form in (4) and different from that in (17):

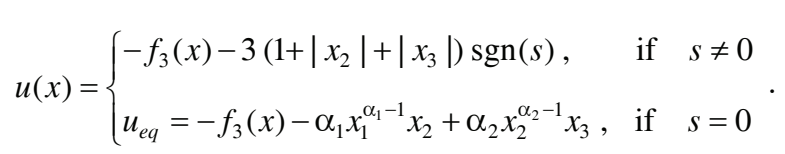

Thus, the system converges to the sliding surface in finite time, and in the sliding surface, the state reaches equilibrium in finite time, which implies finite-time convergence of the closed-loop system. To avoid the singularity (referring to Remark 2), we change (19) to a simple form:

$$
u(x)=\left\{\begin{array}{ll}
-f_{3}(x)-3\left(1+\left|x_{2}\right|+\left|x_{3}\right|\right) \operatorname{sgn}(s), & \text { if } s \neq 0 \\
-f_{3}(x), & \text { if } s=0
\end{array} .\right.
$$

Note that $u=-f_{3}(x)$ cannot keep $s \equiv 0$ when its initial state is already in the sliding surface $S$. Hence, $S$ does not exist in the conventional sense.

\subsection{Class 2}

There are many (smooth) stabilizable nonlinear systems that cannot be stabilized by means of time-invariant smooth feedback. Usually, these nonlinear systems are hard to control effectively, and their variety makes it even harder to obtain a unified method of stabilization. Here, we will only consider a well-known system, but the proposed design idea may also be applicable to generalized systems with similar structures (such as those studied in [12]).

The following well-known smooth system has been widely studied (referring to [12]):

$$
\left\{\begin{array}{l}
\dot{x}_{1}=x_{1}+x_{2}^{3} \\
\dot{x}_{2}=f_{2}\left(x_{1}, x_{2}\right)+u
\end{array} .\right.
$$

This system cannot be stabilized by means of smooth feedback. In this case, conventional terminal sliding mode control design methods may also encounter some difficulties.

The guideline for selecting the switching surface $s=x_{2}-h\left(x_{1}\right)$ for this system is based on the idea given in section 3: the given $h$ makes $\dot{x}_{1}=x_{1}+h^{3}$ finite-time convergent. It is easy to see that we can take $h\left(x_{1}\right)=$ $-\left(x_{1}^{7 / 9}+x_{1}\right)^{1 / 3}$, or equivalently, $s(x)=x_{2}+\left(x_{1}^{7 / 9}+x_{1}\right)^{1 / 3}$. 
Taking $K_{0}=1$ and $L_{0}=2.5$ yields condition (3) by Lemma 1 . Therefore, when $s \neq 0$, the control law can be taken as $u=-3\left(1+\left|x_{1}+x_{2}^{3}\right|\right) \operatorname{sgn}(s)$, according to Theorem 1. On the sliding surface, we can derive

$$
u_{e q}(x)=-f_{2}\left(x_{1}, x_{2}\right)+\frac{x_{1}^{7 / 27}+\frac{7}{9} x_{1}^{1 / 27}}{3\left(1+x_{1}^{2 / 9}\right)^{2 / 3}},
$$

which forces the state to stay in the sliding surface, since it is governed by the dynamics $\dot{x}_{1}=x_{1}+h^{3}=-x_{1}^{7 / 9}$, and it arrives at the origin in finite time. Therefore, a terminal sliding mode controller for system (21) is

$$
u(x)=\left\{\begin{array}{lll}
-3\left(1+\left|x_{1}+x_{2}^{3}\right|\right) \operatorname{sgn}(s), & \text { if } & s \neq 0 \\
u_{e q}(x) \text { in }(22), & \text { if } & s \neq 0
\end{array} .\right.
$$

\section{CONCLUSION}

In the paper, a new design method for terminal sliding mode control has been proposed. Terminal sliding mode controllers have been constructed. They are not in a recursive form, and they can remove singularities outside of sliding surfaces. Future work will focus on developing a complete theory for the construction of these controllers.

\section{ACKNOWLEDGMENT}

The authors wish to thank the anonymous reviewers for their helpful comments on this paper. This work was supported partially by the NNSF of China under Grants 60425307 and 10472129 and partially by the Fund of Returned Overseas Scholars of China.

\section{REFERENCES}

1. Utkin, V., Sliding Modes in Control Optimization, Springer-Verlag, Berlin, Germany (1992).
2. Edwards, C. and S. Spurgeon, Sliding Mode Control, Theory and Applications, Taylor \& Francis, London, U.K. (1998).

3. Cheng, D. and Y. Hong, "Stability of Ideal Sliding Dynamics," Control Theory, Stochastic Analysis and Applications, S. Chen and J. Yong, Eds., World Sci. Publishing, River Edge, NJ, U.S.A., pp. 105118 (1991).

4. Sira-Ramirez, H., "On the Sliding Mode Control of Nonlinear Systems," Syst. Contr. Lett., Vol. 19, No. 3, pp. 303-312 (1992).

5. Yu, X. and Z. Man, "Model Reference Adaptive Control Systems with Terminal Sliding Modes," Int. J. Contr., Vol. 64, No. 4, pp. 1165-1176 (1996).

6. Levant, A., "Universal Single Input Single Output Sliding Mode Controllers with Finite Time Convergence," IEEE Trans. Automat. Contr., Vol. 46, No. 6, pp. 1447-1451 (2001).

7. Man, Z., A. Paplinski, and H. Wu, "A Robust MIMO Terminal Sliding Mode Control for Rigid Robotic Manipulators," IEEE Trans. Automat. Contr., Vol. 39, No. 12, pp. 2464-2469 (1994).

8. Wu, Y., X. Yu, and Z. Man, "Terminal Sliding Mode Control Design for Uncertain Dynamic Systems," Syst. Contr. Lett., Vol. 34, No. 3, pp. 281-287 (1998).

9. Bhat, S. and D. Bernstein, "Finite-Time Stability of Homogeneous Systems," Proc. Amer. Contr. Conf. Albuquerque, NM, U.S.A., pp. 2513-2514 (1997).

10. Hong, Y., "Finite-Time Stabilization and Stabilizability of a Class of Nonlinear Systems," Syst. Contr. Lett., Vol. 46, No. 2, pp. 231-236 (2002).

11. Filippov, A., Differential Equations with Discontinuous Right Hand Side, Kluwer, Dordrecht, The Neitherland (1988).

12. Celikovsky, S. and E. Aranda-Bricaire, "Constructive Nonsmooth Stabilization of Triangular Systems," Syst. Contr. Lett., Vol. 36, No. 1, pp. 21-37 (1999). 